\title{
BMJ
}

\section{Changes in white matter as determinant of global functional decline in older independent outpatients: three year follow- up of LADIS (leukoaraiosis and disability) study cohort}

\begin{abstract}
Domenico Inzitari, full professor of neurology and chairman of the department, ${ }^{1}$ Giovanni Pracucci, neurologist, Anna Poggesi, neurologist PhD student," Giovanna Carlucci, assistant professor in neurology, Frederik Barkhof, full professor of neuroradiology, ${ }^{2}$ Hugues Chabriat, professor of neurology, ${ }^{3}$ Timo Erkinjuntti, full professor of neurology, ${ }^{4}$ Franz Fazekas, full professor of neurology, ${ }^{5}$ José M Ferro, chairman professor of neurology, ${ }^{6}$ Michael Hennerici, full professor of neurology and chairman of the department, ${ }^{7}$ Peter Langhorne, full professor of geriatric medicine, ${ }^{8}$ John O'Brien, professor of old age psychiatry, ${ }^{9}$ Philip Scheltens, full professor of neurology, ${ }^{2}$ Marieke C Visser, assistant professor of neurology, ${ }^{2}$ Lars-Olof Wahlund, professor of geriatric medicine, ${ }^{10}$ Gunhild Waldemar, full professor of neurology,,$^{11}$ Anders Wallin, full professor of neuropsychiatry, ${ }^{12}$ Leonardo Pantoni, consultant neurologist ${ }^{1}$ on behalf of the LADIS Study Group
\end{abstract}

${ }^{1}$ Department of Neurological and Psychiatric Sciences, University of Florence, Viale Morgagni 85, 50134 Firenze, Italy

${ }^{2}$ Department of Radiology and Neurology, VU Medical Centre, Amsterdam, Netherlands

${ }^{3}$ Department of Neurology, Hopital Lariboisiere, Paris, France

${ }^{4}$ Memory Research Unit,

Department of Clinical

Neurosciences, Helsinki

University, Helsinki, Finland

${ }^{5}$ Department of Neurology and $M$

RI Institute, Medical University

Graz, Austria

${ }^{6}$ Serviço de Neurologia, Centro de

Estudos Egas Moniz, Hospital de

Santa Maria Lisboa, Portugal

${ }^{7}$ Department of Neurology,

University of Heidelberg, Klinikum Mannheim, Mannheim, Germany

${ }^{8}$ Academic Department for

Geriatric Medicine, Glasgow Royal

Infirmary, Glasgow

${ }^{9}$ Institute for Ageing and Health, Newcastle University, Newcastle upon Tyne

${ }^{10}$ Karolinska Institute, Department of Neurobiology, Care Sciences and Society, Karolinska University Hospital Huddinge, Huddinge,

Sweden

${ }^{11}$ Memory Disorders Research

Unit, Department of Neurology,

Copenhagen University Hospital,

Copenhagen, Denmark

${ }^{12}$ Institute of Clinical

Neuroscience, Gothenburg

University, Gothenburg, Sweden

Correspondence to: D Inzitari

inzitari@neuro.unifi.it

Cite this as: BMJ 2009;339:b2477 doi:10.1136/bmi.b2477

\section{ABSTRACT}

Objective To assess the impairment in daily living activities in older people with age related changes in white matter according to the severity of these changes. Design Observational data collection and follow-up of a cohort of older people undergoing brain magnetic resonance imaging after non-disabling complaints.

Setting 11 European centres.

Participants 639 non-disabled older patients (mean age 74.1 (SD 5.0), 45.1\% men) in whom brain magnetic resonance imaging showed mild, moderate, or severe age related changes in white matter (Fazekas scale). Magnetic resonance imaging assessment also included cerebral infarcts and atrophy.

Main outcome measure Transition from no disability (defined as a score of 0 or 1 on the instrumental activities of daily living scale) to disability (score $\geq 2$ ) or death over three year follow-up. Secondary outcomes were incident dementia and stroke.

Results Over a mean follow-up period of 2.42 years (SD 0.97 , median 2.94 years), information on the main outcome was available for 633 patients. The annual rate of transition or death was $10.5 \%, 15.1 \%$, and $29.5 \%$, respectively, for patients with mild, moderate, or severe age related changes in white matter (Kaplan-Meier log rank test P 0.001). In a Cox model comparing severe with mild changes and adjusted for clinical factors of functional decline, the risk of transition to disability or death was more than twofold higher (hazard ratio 2.36, $95 \%$ confidence interval 1.65 to 3.81 ). The other predictors were age group, history of atrial fibrillation, and complaint of gait disturbances. The effect of severe changes remained significant independently of baseline degree of atrophy and number of infarcts. Incident stroke and dementia only slightly modified this effect.
Conclusion The three year results of the LADIS study suggest that in older adults who seek medical attention for non-disabling complaints, severe age related changes in white matter independently and strongly predict rapid global functional decline.

\section{INTRODUCTION}

Many older people have age related changes in white matter on brain magnetic resonance imaging. Such changes, also called leukoaraiosis when detected by computed tomography ${ }^{1}$, appear as hyperintense areas seated in the white matter on $\mathrm{T} 2$ weighted magnetic resonance imaging sequences, from mild focal to large confluent, in about a third of cases of moderate to severe grade. ${ }^{2}$

Cognitive impairment, depression, difficulties in walking, and urinary incontinence are the main contributors to loss of functional independence in older people. All the above have been consistently reported to be associated with age related changes in white matter with a potential impact on global functioning. ${ }^{3}$ There have been no studies of adequate power primarily aimed at assessing such changes and their severity in relation to daily living disability. Scarce evidence exists from one study that examined a small sample of patients with computed tomography defined leukoaraiosis compared with controls without leukoaraiosis ${ }^{4}$ and from two studies in subsets of patients with selective clinical presentations (older patients with major depression, ${ }^{5}$ patients with subcortical vascular dementia $\left.^{6}\right)$.

The LADIS (leukoaraiosis and disability) study is a multicentre European collaboration that started in 2000 to investigate, through a longitudinal design, whether age related changes in white matter are an 
independent determinant of functional decline in older people. Age related pathological processes involved in the transition from independent functional status to more substantial disability have not been completely elucidated. Modern laboratory techniques (for example, brain imaging) might help to identify these processes. Both prevalence and severity of age related changes in white matter increase with age, and several dysfunctions occurring with age and contributing to disability have been reported to be associated with such changes, raising the hypothesis that they might be one of the processes causing disability in older people. We need to establish their net contribution in relation to other factors of disability. We do not know what grade of changes produces functional effects and whether there is a dose-effect or a threshold relation between the severity of changes and the risk of functional decline.

We present the three year follow-up results of the main prespecified outcome - that is, the transition (in relation to the baseline grade of age related changes in white matter) from independence in the instrumental activities of daily living to disability or death, as evidenced in a cohort of older people undergoing brain magnetic resonance imaging after non-disabling complaints.

\section{METHODS}

Full details of the LADIS study are reported elsewhere. $^{7}$

\section{End point definition}

Our primary end point was transition to disability, based on Lawton and Brody's instrumental activity of daily living scale ${ }^{8}$ as the change from a score of 0 or 1 at baseline to a score of 2 or more or death. Our definition of transition corresponds with the progression from no or mild to moderate or severe disability and is the categorisation used by longitudinal population based studies of disability conducted in similar aged cohorts. ${ }^{9-11}$ We used the risks of such transition plus death (as estimated by these studies) to calculate power and sample size. ${ }^{7}$ Two population based studies reported the risk of the transition from no or mild to moderate or severe disability to be $25 \%$ after three year follow-up in people aged 65-84. ${ }^{112}$ Assuming that the group with the mildest degree of age related changes in white matter has a

Table 1|Reasons for referral of people enrolled in LADIS study and distribution according to severity of age related changes in white matter. Figures are numbers (percentages) of participants

\begin{tabular}{lcccc} 
& $\begin{array}{c}\text { Mild } \\
(\mathrm{n}=\mathbf{2 8 2})\end{array}$ & $\begin{array}{c}\text { Moderate } \\
(\mathrm{n}=196)\end{array}$ & $\begin{array}{c}\text { Severe } \\
(\mathrm{n}=\mathbf{1 5 5 )}\end{array}$ & $\begin{array}{c}\text { Total } \\
(\mathrm{n}=633)\end{array}$ \\
$\begin{array}{l}\text { Mild memory or motor problems, } \\
\text { mood alterations }\end{array}$ & $88(42.3)$ & $71(34.1)$ & $49(23.6)$ & $208(100)$ \\
\hline Minor cerebrovascular events & $31(25.6)$ & $42(34.7)$ & $48(39.7)$ & $121(100)$ \\
\hline Incidental computed tomography or MRI finding & $56(52.8)$ & $28(26.4)$ & $22(20.8)$ & $106(100)$ \\
\hline Other neurological symptoms & $55(43.3)$ & $43(33.9)$ & $29(22.8)$ & $127(100)$ \\
\hline Other study controls, volunteers & $52(73.2)$ & $12(16.9)$ & $7(9.9)$ & $71(100)$ \\
\hline MRI=magnetic resonance imaging. & & & & \\
\hline
\end{tabular}

$25 \%$ risk of transition to disability or death over three years (equal to that of the general older population), we needed 800 people to show a significant trend (1.7 estimated relative risk) in the effect of exposure to the three increasing degrees of severity, with adjustment for age, reasons for presentation, and comorbidity ( $\alpha$ error probability $5 \%$ (two tailed), power $80 \%$ ). As recruitment proved to be slower than expected, we revised the original estimation - that is, while it was approaching 600 patients. From a preliminary baseline data analysis, the participants with severe age related changes in white matter, although comparable on instrumental activities of daily living and on most risk factors with the patients in the other two groups, turned out to have considerably greater motor and cognitive impairment than expected, even after adjustment for several other predictors of disability. We therefore hypothesised that those in the group with severe changes were more prone to progress to disability than expected, and the relative risk among the group with severe changes compared with the milder changes could be greater than expected (relative risk 1.7). With a relative risk of around 2.0, we recalculated that we needed 500 patients to show a significant effect of the increasing severity of age related changes in white matter on the risk of transition. Taking into account a probable dropout rate of around $20 \%$, we considered it sufficient to include 650 participants. The final sample, after checking for completeness of data, was 639.

Secondary outcomes in the LADIS study were incident dementia and stroke, according to the Diagnostic and Statistical Manual of Mental Disorders, fourth edition $^{13}$ and World Health Organization criteria, respectively. ${ }^{14}$

\section{Patients}

Participants were patients aged 65-84 in whom age related changes in white matter of any degree were found when they were being investigated at one of the 11 collaborating European centres for complaints such as mild memory or motor problems, minor cerebrovascular events (non-disabling strokes or transient ischaemic attacks), mood alterations, and other minor neurological problems, none interfering with common activities of daily living. Those with incidentally discovered age related changes in white matter were also considered. Reasons for referral were grouped into cognitive, motor or mood complaints; non-disabling strokes or transient ischaemic attacks; other neurological symptoms; incidental findings on computed tomography or magnetic resonance imaging; and participants in a population survey. Table 1 shows numbers of individuals in each category and their subdivision according to grade of changes. To be included, patients had to perform without help in every activity of the instrumental activities of daily living scale or had to be limited in only one activity. Other inclusion criteria were signed informed consent and the availability of a regularly contactable informant. The informant was a next of kin or a close friend of the patient supposedly contactable during follow-up. 
Reasons for exclusion were lack of age related changes in white matter, severe illnesses (cardiac, hepatic, or renal failure, cancer or other relevant systemic diseases), unrelated neurological diseases, leukoencephalopathy of non-vascular origin (immunologicaldemyelinating, metabolic, toxic, infectious, other), and severe psychiatric disorders.

Out of the 897 consecutive patients screened for inclusion, $180(20 \%)$ were excluded because wrong age $(n=76)$, disability or presence of severe illnesses possibly interfering with follow-up assessment $(\mathrm{n}=57)$, absence of age related white matter changes $(n=27)$, and inability or refusal to undergo magnetic resonance imaging scanning $(n=20)$, thus leaving 717 eligible patients. Seventy eight of the eligible patients refused to participate, and 639 (71\% of the screened sample) were finally enrolled in the study.

\section{Assessment}

Entry assessment included a detailed magnetic resonance imaging study and several functional and clinical measures. ${ }^{7}$ Patients were then asked to undergo yearly clinical and functional assessments for a maximum of three years. At entry and at each follow-up visit, a structured questionnaire was used to assess, among other variables, education (expressed as years of schooling), living conditions (alone or with others), history of myocardial infarction and angina pectoris (ischaemic heart disease), stroke, heart failure, arrhythmias, arterial hypertension, peripheral vascular disease, diabetes mellitus, chronic obstructive pulmonary disease, depression, gait disturbances, osteoarthritis, falls in the past year, visual impairment, and hearing loss. The definition of each variable was based on recent criteria. ${ }^{7}$ All the patients underwent a standard physical examination during which body mass index and pulse pressure were also recorded.

The instrumental activities of daily living scale includes eight items: ability to use the telephone, shopping, food preparation, housekeeping, laundry, mode of transportation, responsibilities for own medications, and ability to handle finances. ${ }^{8}$ Depending on the type of task, questions were asked as to whether the task was accomplished without any limitation, with difficulties, or with help. Our outcome variable was no limitation versus limitation of any degree (either difficulty or need for help) in one or more of the tasks included in the scale. The IADL scale was administered from baseline only to the informant because of the expectation of cognitive decline at follow-up. The reliability of scoring between raters and centres, tested in advance, showed good agreement on each item rating ( $\kappa$ ranging from 0.69 to 0.85 ). Functional status was also assessed with the informant for patients who had died (assessment related to functional status before death) or had refused to undergo a follow-up visit. The date (month) of status change was registered.

All participants underwent magnetic resonance imaging following a standard protocol, during which $0.5 \mathrm{~T}$ or $1.5 \mathrm{~T}$ scanners were used and series included axial T2 weighted images (echo time 100-120 ms; repetition time $4000-6000 \mathrm{~ms}$; voxel size $1 \times 1 \times 5-7.5 \mathrm{~mm}^{3} ; 19-24$ slices), axial fluid attenuated inversion recovery images (echo time 100-140 ms; repetition time 6000$10000 \mathrm{~ms}$; inversion time $2000-2400 \mathrm{~ms}$; voxel size $1 \times 1 \times 5-7.5 \mathrm{~mm}^{3} ; 19-24$ slices $)$, and coronal or sagittal 3D T1 sequence (TE 4-7 ms; TR 10-25 ms; flip angle $15-30^{\circ}$; voxel size $\left.1 \times 1 \times 1-1.5 \mathrm{~mm}^{3}\right)$. All scans were checked and stored at the image analysis centre of the VU Medical Centre, Amsterdam, Netherlands.

A central rater who was blind to the clinical and functional data used the visual Fazekas scale to rate severity of age related changes in white matter at baseline on magnetic resonance imaging. ${ }^{15}$ Accordingly, patients were subdivided into three severity groups: grade 1 (mild changes): single lesions $<10 \mathrm{~mm}$ and/or areas of "grouped" lesions $<20 \mathrm{~mm}$ in any diameter; grade 2 (moderate changes): single hyperintense lesions 10-

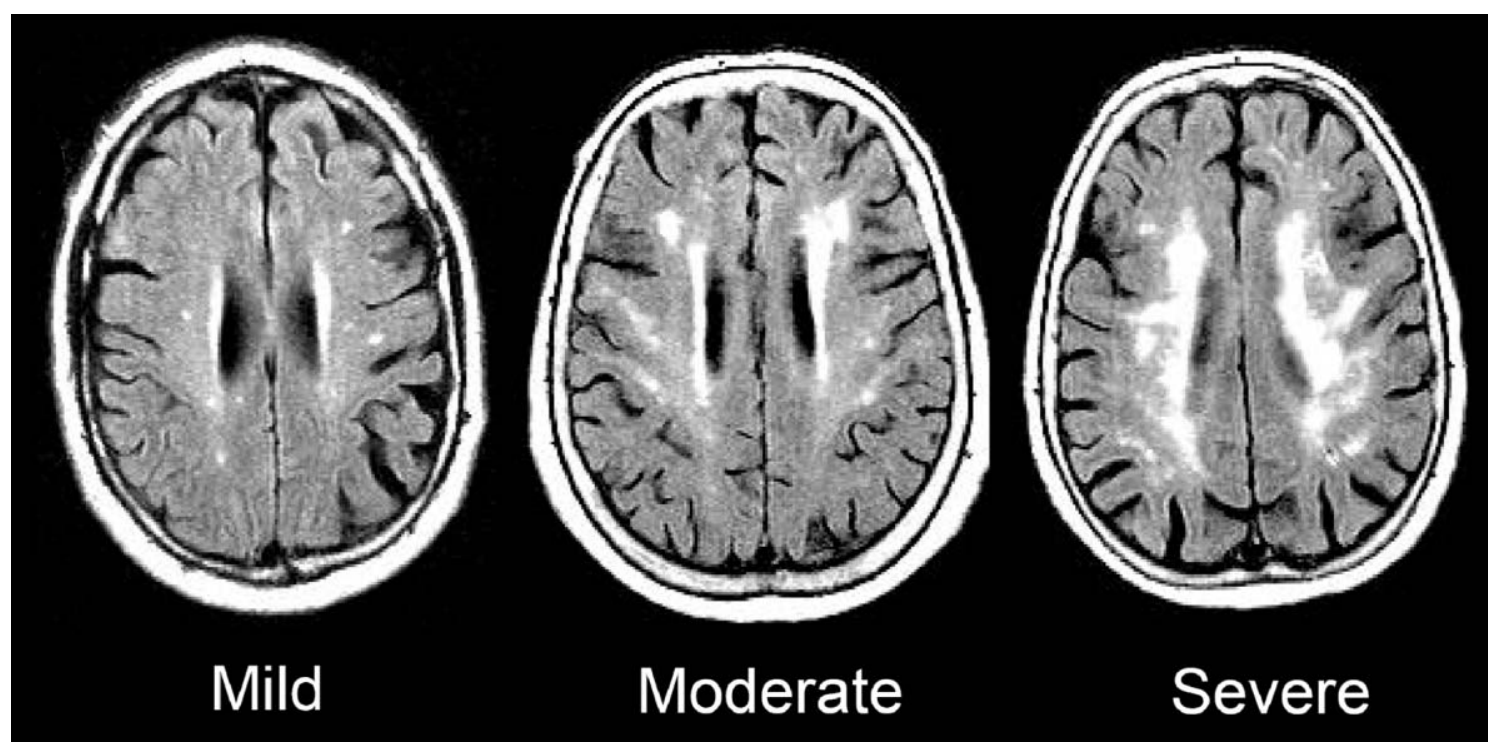

Fig 1 Age related changes in white matter: three severity grades according to modified Fazekas scale 
Table 2 |Demographics and functional status according to severity of age related changes in white matter (Fazekas scale) (633 patients with follow-up assessment). Figures are numbers (percentages) of participants unless stated otherwise

\begin{tabular}{|c|c|c|c|c|c|}
\hline & Mild $(n=282)$ & Moderate $(n=196)$ & Severe $(n=155)$ & Total $(n=633)$ & $P$ value \\
\hline Sex (male) & $128(45.4)$ & $76(38.8)$ & $81(52.3)$ & $285(45.0)$ & 0.314 \\
\hline \multicolumn{6}{|l|}{ Age (years): } \\
\hline $65-69$ & $85(30.1)$ & $46(23.5)$ & $27(17.4)$ & $158(25.0)$ & \multirow{4}{*}{0.001} \\
\hline $70-74$ & $93(33.0)$ & $58(29.6)$ & $49(31.6)$ & $200(31.6)$ & \\
\hline $75-79$ & $70(24.8)$ & $58(29.6)$ & $54(34.8)$ & $182(28.7)$ & \\
\hline $80-84$ & $34(12.1)$ & $34(17.3)$ & $25(16.1)$ & $93(14.7)$ & \\
\hline Living condition (with others) & $176(62.4)$ & $109(55.6)$ & $104(67.1)$ & 389 (61.5) & 0.541 \\
\hline Mean (SD) age (years) & $73.3(5.0)$ & $74.4(5.1)$ & $75.0(4.8)$ & $74.1(5.0)$ & 0.001 \\
\hline Mean (SD) education (years of schooling)* & $9.8(3.6)$ & $9.6(3.9)$ & $9.3(4.0)$ & $9.6(3.8)$ & 0.129 \\
\hline $\begin{array}{l}\text { Score } 0 v 1 \text { on instrumental activities } \\
\text { of daily living at baseline }\end{array}$ & $36(12.8)$ & $41(20.9)$ & $42(27.1)$ & $119(18.8)$ & $<0.001$ \\
\hline
\end{tabular}

$20 \mathrm{~mm}$, and hyperintense areas linked by no more than "connecting bridges" >20 $\mathrm{mm}$ in any diameter; and grade 3 (severe changes): both single and confluent hyperintense areas of $\geq 20 \mathrm{~mm}$ in any diameter) (fig 1). Volumetric assessment was performed on a Sparc 5 workstation (SUN, Palo Alto, CA). A single rater used a semi-automated method to measure the volume of white matter changes on axial fluid attenuation inversion recovery images, both in supratentorial and infratentorial regions. Lesions were marked and borders were set with local thresholding (home developed software Show_Images, version 3.6.1) on each slice. No distinction was made between subcortical and periventricular hyperintensities. ${ }^{16}$

The LADIS study also assessed several magnetic resonance imaging variables. As possible confounders of outcome in our study, we used lacunar infarcts defined as hypointense foci of $\geq 3 \mathrm{~mm}$ on $\mathrm{T} 1$ sequences that were surrounded by white matter or subcortical grey matter and not located in areas with a high prevalence of widened perivascular spaces (for example, anterior commissure, vertex), the number of lacunes being recoded into none, few (1-3), and many $(\geq 4)^{17}$; non-lacunar infarcts dichotomised as none/at least one; and global atrophy, rated on fluid attenuation inversion recovery scans, for ventricular and sulcal widening separately, using a template based rating scale ranging from 1 (no) to 8 (severe) atrophy. The inter-rater $\kappa$ was 0.70 for sulcal atrophy and 0.83 for ventricular atrophy. ${ }^{18}$ Measures of lacunar infarcts, non-lacunar infarcts, and atrophy were not available for two, three, and 109 patients, respectively.

\section{Statistical analysis}

In the 633 patients followed up, we first examined variations of baseline demographics, risk factors, and comorbidities across the three severity groups, assessing significance by means of $\chi^{2}$ test for trend for categorical variables and trend test (analysis of variance with polynomial contrast) for continuous variables. Results are reported as frequency and percentage or mean and standard deviation.

We expressed the incidence rate of transition to disability or death (primary end point) and stroke and dementia (secondary end points) in each of the three severity groups (Fazekas rating) as number of events per 100 person years at risk, with $95 \%$ confidence intervals.

The Kaplan-Meier curves examined the probability of surviving free of disability or death across the

Table 3 |Vascular risk factors and comorbidities according to severity of age related changes in white matter (Fazekas scale) groups (633 patients with follow-up assessment*). Figures are numbers (percentages) of participants unless stated otherwise

\begin{tabular}{|c|c|c|c|c|c|}
\hline & Mild $(n=282)$ & Moderate $(n=196)$ & Severe $(n=155)$ & Total $(n=633)$ & $P$ value \\
\hline Ischaemic heart disease & $57(20.4)$ & $40(20.4)$ & $30(19.5)$ & $127(20.2)$ & 0.828 \\
\hline Heart failure & $7(2.5)$ & $5(2.6)$ & $9(5.8)$ & $21(3.3)$ & 0.086 \\
\hline Arterial hypertension & $179(63.7)$ & $145(74.0)$ & $118(76.6)$ & $442(70.0)$ & 0.003 \\
\hline Atrial fibrillation & $21(7.5)$ & $13(6.7)$ & $15(9.7)$ & $49(7.8)$ & 0.475 \\
\hline Diabetes & $33(11.7)$ & 29 (14.9) & $27(17.8)$ & 89 (14.1) & 0.078 \\
\hline History of stroke & $49(17.4)$ & $61(31.3)$ & $73(47.1)$ & $183(28.6)$ & $<0.001$ \\
\hline Peripheral vascular disease & $19(6.8)$ & $16(8.2)$ & $10(6.6)$ & $45(7.2)$ & 0.952 \\
\hline Mean (SD) BMI & $26.1(4.2)$ & $26.1(4.1)$ & $26.4(4.5)$ & $26.2(4.2)$ & 0.533 \\
\hline Mean (SD) pulse pressure & $64.3(16.9)$ & $66.8(16.4)$ & $66.8(15.6)$ & $65.7(16.5)$ & 0.134 \\
\hline
\end{tabular}

*Data missing for ischaemic heart disease (4), heart failure (2), arterial hypertension (2), atrial fibrillation (3), diabetes (4), history of stroke (1), peripheral vascular disease (5). 
Table $4 \mid$ Other disabling comorbidities according to severity of age related changes in white matter (Fazekas scale) (633 patients with follow-up assessment*). Figures are numbers (percentages) of participants

\begin{tabular}{|c|c|c|c|c|c|}
\hline & Mild $(n=282)$ & Moderate $(n=196)$ & Severe $(n=155)$ & Total $(n=633)$ & $P$ value \\
\hline Depression & $78(27.7)$ & $58(29.6)$ & $39(25.2)$ & $175(27.6)$ & 0.668 \\
\hline Falls in the last year & $63(22.3)$ & $61(31.3)$ & $57(36.8)$ & $181(28.6)$ & 0.001 \\
\hline Osteoarthritis & $82(29.2)$ & $56(28.7)$ & $39(25.3)$ & $177(28.1)$ & 0.421 \\
\hline Chronic obstructive pulmonary disease & $27(9.6)$ & $18(9.2)$ & $20(12.9)$ & $65(10.3)$ & 0.326 \\
\hline Complaint of gait disturbances & $100(35.8)$ & $74(38.1)$ & $83(54.6)$ & $257(41.1)$ & $<0.001$ \\
\hline Urinary disturbances & $110(39.0)$ & $68(34.7)$ & $75(48.4)$ & $253(40.0)$ & 0.113 \\
\hline Visual impairment & $37(13.1)$ & $35(17.9)$ & $39(25.2)$ & $111(17.5)$ & 0.002 \\
\hline Hearing loss & $124(44.0)$ & $89(45.4)$ & $73(47.1)$ & $286(45.2)$ & 0.528 \\
\hline
\end{tabular}

*Data missing for falls in past year (1), osteoarthritis (3), chronic obstructive pulmonary disease (1), complaint of gait disturbances (8).

severity groups (separated by either the three Fazekas ranks or volume fifths). We estimated differences between groups with the log rank test. Median survival time in years was also reported.

With the mild group taken as reference, ${ }^{7}$ we compared the net predictive effect of Fazekas grades on the primary end point in the severe and moderate groups with Cox regression analysis, controlling for the several potential confounders listed in the assessment section. In addition to demographic factors, these included the factors resulting as the best predictors of decline in global function among older people in the meta-analysis of Stuck et al. ${ }^{19}$ Because patients were enrolled in different centres, we also adjusted for this factor. In addition, we entered the baseline score for instrumental activities of daily living ( 0 or 1 ). Finally, to properly control for the different conditions that had led patients to be included in the study, we used a Cox model stratified by the reasons for referral (table 1). In a further model, we controlled for the effect of age related changes in white matter on transition, including magnetic resonance imaging infarcts and atrophy.

During the follow-up period 242 patients reached the primary end point and 391 did not and were therefore censored (that is, were not counted in estimating the risk of the outcome). Of these 391 censored patients, 331 had completed the scheduled follow-up and were considered as non-informative regarding the outcome. The 60 remaining patients, who had been observed for shorter periods, were retained as still potentially informative. Baseline factors did not differ significantly between these two groups.

Data were analysed with SPSS version 14.

Table 5 |Rate (95\% confidence interval) per 100 person years at risk of composite outcome (transition to disability or death) and of individual outcomes according to severity of age related changes in white matter

Mild Moderate Severe Total sample

Transition to disability $\quad 10.5$ (8.2 to 12.8$) \quad 15.1$ (11.6 to 18.6$) \quad 29.5(23.5$ to 35.5$) \quad 15.8$ (13.8 to 17.8$)$ or death

Transition to disability $\quad 9.1(6.9$ to 11.2$) \quad 13.7(10.4$ to 17.0$) \quad 27.9(22.1$ to 33.8$) \quad 14.4(12.5$ to 16.3$)$

Death $\quad 2.1(1.1$ to 3.1$) \quad 1.8(0.7$ to 2.9$) \quad 5.4(1.8$ to 5.4$) \quad 2.4$ (1.7 to 3.1$)$

\section{RESULTS}

At follow-up, of the 639 patients (mean age 74.1 (SD $5.0)$ years, $45.1 \%$ men) enrolled, we had information about vital and instrumental activities of daily living status for $633(99.1 \%)$ (mean age 74.1 (SD 5.0) years, $45.0 \%$ men). Among these patients, according to the three Fazekas grades on baseline magnetic resonance imaging scan, $44 \%$ had mild, $31 \%$ had moderate, and $25 \%$ had severe age related changes in white matter. In three groups compared by clinical and functional features at entry, we found that older age, history of arterial hypertension, stroke, falls in the past year, complaint of gait disturbances, and visual problems were all more common among patients with the severe grade (tables 2, 3, and 4).

Over a mean follow-up period of 2.42 years (SD 0.97, median 2.94 years), 242 (38.2\%, mean age 75.4 (SD 4.8), 44.2\% men) of the 633 patients reached the study end point. Table 5 shows the frequency of the composite primary outcome (transition to disability or death) and of individual outcomes. Patients with severe age related changes in white matter had a worse prognosis; about $60 \%$ had become disabled or had died at the end of the follow-up.

The rate (per 100 person years at risk) of transition from independence in instrumental activities of daily living activities to disability or death was $10.5(95 \%$ confidence interval 8.2 to 12.8 ), 15.1 (11.6 to 18.6), and 29.5 (23.5 to 35.5) for patients in the mild, moderate, and severe Fazekas scale group, respectively. Survival analysis carried out for each of the Fazekas scale grades or volume fifths (fig 2) showed that the probability of surviving free of disability or death decreased with the increasing severity of changes in white matter (Kaplan-Meier log rank test $\mathrm{P}<0.001$ for either grading). When we compared the moderate with the severe group, and the fourth with the fifth group, median survival times were 3.27 (3.16 to 3.38 ) v 2.14 (1.67 to 2.62) and 3.27 (3.12 to 3.42$)$ v 2.09 (1.72 to 2.47 ) years, respectively.

The rate (per 100 person years at risk) of transition from independence in instrumental activities of daily living activities to disability or death varied according to the reason for referral (table 6). Patients with mild memory or motor problems and mood alteration or 

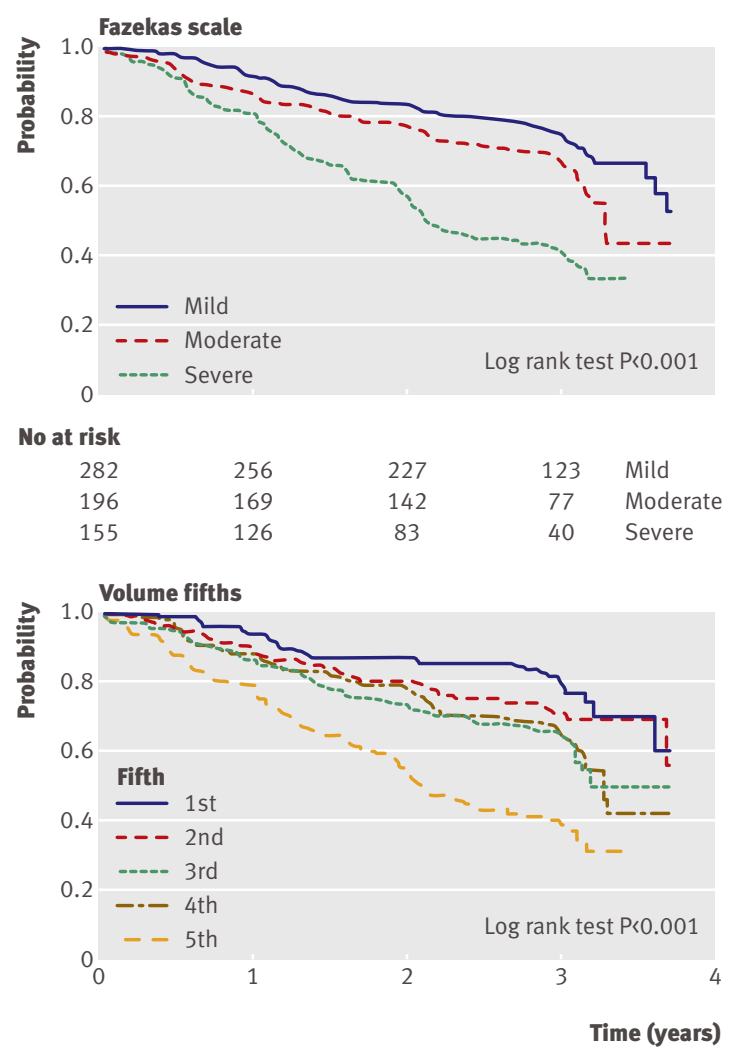

No at risk

$\begin{array}{lcccc}122 & 113 & 103 & 51 & 1 \text { st } \\ 122 & 108 & 91 & 51 & \text { 2nd } \\ 122 & 104 & 85 & 46 & 3 \text { rd } \\ 123 & 109 & 91 & 56 & \text { 4th } \\ 120 & 95 & 64 & 28 & 5 \text { th }\end{array}$

Fig 2 | Kaplan-Meier probability of survival free of transition from independence in instrumental activities daily living to disability or death according to baseline severity grades in age related changes in white matter

minor cerebrovascular events had the worst prognosis, whereas those enrolled as other study controls or volunteers the best prognosis. This basically parallels the different distribution of severe age related changes in white matter across reason for referral groups.

When we compared the severe with the mild grades of changes and adjusted for confounders by Cox analysis (table 7), the hazard of transition to disability or death was more than twofold higher for the severe Fazekas group. Other factors that independently predicted transition or death were age group, history of atrial fibrillation, complaint of gait disturbances, and baseline instrumental activities of daily living score of 1. Education and history of osteoarthritis were protective factors (table 7).

Patients with moderate age related changes in white matter also had a significantly higher risk of transition or death in the unadjusted comparison with the mild group (hazard ratio $1.48,1.08$ to 2.05 ), but the difference was no longer significant after adjustment (table 7 ). The attenuation turned out to depend mostly on the effect of age. When we analysed fifths of volume of age related changes in white matter with adjustment for confounders, the hazard of transition to disability or death was almost threefold higher (hazard ratio $2.99,1.77$ to 5.05 ) comparing the highest fifth with the lowest.

When we considered as outcome the transition to disability alone (excluding death), the adjusted hazard ratios were 1.09 (0.74 to 1.59$)$ for patients with moderate changes and 2.50 (1.72 to 3.65) for those with severe changes, in comparison with the mild group.

Evidence of multiple lacunar infarcts and non-lacunar infarcts and severity of atrophy on magnetic resonance imaging at baseline was also significantly associated with the degree of severity of age related changes in white matter (table 8). When we added these variables to the Cox model, together with the three severity grades and all other confounders, severe changes remained an independent predictor of transition to disability or death (hazard ratio $2.36,1.55$ to 3.60).

Severity grades of age related changes in white matter independently predicted the severity of functional decline as expressed by the mean number of instrumental activities of daily living changed by follow-up (1.09 (SD 1.99) in the mild, 1.42 (SD 2.0) in the moderate, and 2.53 (SD 2.69) in the severe group; $\mathrm{P}<0.001$ after adjustment for confounders). The activities most commonly limited among patients with functional decline were housekeeping, shopping, and mode of transportation

In the mild, moderate, and severe change groups, the rate (per 100 person years at risk) of incident stroke was 1.8 (95\% confidence interval 0.8 to 2.8$), 3.2$ (1.6 to 4.9 ), and 6.9 (4.0 to 9.8), and the rate of incident dementia was 3.2 (1.9 to 4.5 ), 4.7 (2.8 to 6.6 ), and 12.8 (8.9 to 16.6), respectively. Adding incident stroke and dementia to the Cox regression model only slightly attenuated the effect of severe changes on transition to disability or death (hazard ratio $2.06,1.40$ to 3.04).

Table $6 \mid$ Rate (95\% confidence interval) per 100 person years at risk of transition to disability or death according to reason for referral and severity of age related changes in white matter

\begin{tabular}{lcccc} 
Reasons for referral & Mild & Moderate & Severe & Total \\
Mild memory or motor problems, mood alterations & $15.8(10.5$ to 21.0$)$ & $21.6(14.3$ to 28.8$)$ & $38.1(25.3$ to 50.9$)$ & $22.0(17.7$ to 26.2$)$ \\
\hline Minor cerebrovascular events & $7.2(1.4$ to 12.9$)$ & $13.4(6.6$ to 20.2$)$ & $32.4(20.8$ to 43.9$)$ & $17.7(12.8$ to 22.6$)$ \\
\hline Incidental computed tomography/MRI finding & $7.0(2.8$ to 11.1$)$ & $11.3(3.5$ to 19.2 & $28.2(12.9$ to 43.6$)$ & $11.7(7.6$ to 15.7$)$ \\
\hline Other neurological symptoms & $10.2(5.0$ to 15.3$)$ & $10.9(4.7$ to 17.0$)$ & $19.8(9.0$ to 30.5$)$ & $12.4(8.5$ to 16.2$)$ \\
\hline Other study controls, volunteers & $8.4(3.4$ to 13.4$)$ & $11.7(0$ to 24.9$)$ & $11.6(0$ to 27.7$)$ & $9.2(4.7$ to 13.7$)$ \\
\hline MRI=magnetic resonance imaging. & & &
\end{tabular}


Table $7 \mid$ Effect of severity of age related changes in white matter and other independent predictors of transition from independence in instrumental activities daily living to disability or death*

Hazard ratio ( $95 \%$ confidence interval)

Unadjusted Adjusted $\dagger$

Fazekas grade:

\begin{tabular}{lcc}
\hline Mild & 1 & 1 \\
\hline Moderate & $1.48(1.08$ to 2.05$)$ & $1.13(0.79$ to 1.61$)$ \\
\hline Severe & $3.01(2.22$ to 4.08$)$ & $2.36(1.65$ to 3.81$)$ \\
\hline
\end{tabular}

Age group (years):

\begin{tabular}{|ccc}
\hline $65-69$ & 1 & 1 \\
\hline $70-74$ & $1.77(1.20$ to 2.62$)$ & $1.73(1.14$ to 2.64$)$ \\
\hline $75-79$ & $2.19(1.49$ to 3.22$)$ & $1.91(1.23$ to 2.97$)$ \\
\hline $80-84$ & $2.61(1.71$ to 3.99$)$ & $2.24(1.35$ to 3.70$)$ \\
\hline Baseline instrumental activities of daily living=1 & $3.69(2.83$ to 4.81$)$ & $3.08(2.24$ to 4.23$)$ \\
\hline Atrial fibrillation & $2.04(1.38$ to 3.01$)$ & $1.69(1.09$ to 2.64$)$ \\
\hline Complaint of gait disturbances & $1.97(1.52$ to 2.54$)$ & $1.62(1.16$ to 2.52$)$ \\
\hline History of osteoarthritis & $0.80(0.60$ to 1.08$)$ & $0.58(0.40$ to 0.83$)$ \\
\hline Education (per year of schooling) & $0.94(0.91$ to 0.97$)$ & $0.90(0.86$ to 0.94$)$ \\
\hline
\end{tabular}

*Variables in models were age, sex, education, baseline instrumental activities of daily living $(0 \vee 1)$, centre, living condition, body mass index, pulse pressure, history of ischaemic heart disease, heart failure,

hypertension, atrial fibrillation, diabetes, depression, falls, osteoarthritis, stroke, chronic obstructive pulmonary disease, peripheral vascular disease, gait, urinary, visual, or hearing disturbances. Analysis stratified for reason for referral.

†Cox regression models, adjusted for multiple factors of disability.

\section{DISCUSSION}

\section{Principal findings}

The final results of the LADIS study show that in an older person undergoing brain magnetic resonance imaging after a non-disabling complaint, the presence of severe age related changes in white matter, measured either visually or volumetrically, predicts a more rapid decline in global functioning, independent of age and several factors known to be associated with disability in older people. This effect does not seem to be related to baseline cerebral atrophy or infarcts, both known to be associated with age related changes in white matter, nor is it consequent to incident strokes, despite age related changes in white matter being considered as a stroke marker. ${ }^{20}$ Part of the effect is explained by incident dementia, but the influence of severe changes on disability does not depend exclusively on their ability to determine dementia. The role of severe changes as a determinant of rapid functional decline was already evident after an interim analysis carried out at the one year follow-up. ${ }^{21}$ Concerning the hypothesised dose-effect relation between severity grades and the probability of transition or death, while the predicted number of instrumental activities of daily living impaired would support the hypothesis, the analysis of primary outcome by fifths of volume of changes, showing the remarkable increased risk for the highest fifth, corroborates the hypothesis of a threshold effect.

\section{Strength and limitations of the study}

A main limitation to the generalisability of our results is related to the hospital based design and the recruitment of patients from several different clinical centres, both possible sources of bias linked with referral and selection. Adjustment for reason for referral and for centre did not alter the strength of the effect of age related changes in white matter on progression to disability. Statistical adjustment, however, might not have been enough to fully overcome the referral problem. Nearly all patients were enrolled after seeking medical attention and therefore no inference can be made to subjects with age related white matter changes who are free of symptoms. The reasons for referral that led patients to be included in our study were those commonly leading to discovery of age related changes in white matter in older people, and thus the LADIS sample simply reflects the population of patients with such changes likely to be encountered in clinical practice. Compared with a population based approach, strengths of a clinical study in this setting include an adequate number of patients in each severity group, the detailed and accurate functional and magnetic resonance imaging assessments, and the low dropout rate.

As in previous studies, we used the instrumental activities of daily living scale. The reliability and validity of such a scale is considered sufficient to warrant its use in clinical situations. ${ }^{22}$ Data might be subject to variability depending on sociodemographic factors. ${ }^{23}$ Our data on agreement between centres show that the assessment of instrumental activities of daily living was consistent across several linguistic and sociocultural settings throughout Europe.

Table 8 | Infarcts and atrophy on magnetic resonance imaging according to severity of age related changes in white matter (Fazekas scale) (633 patients with follow-up assessment*). Figures are numbers (percentages) of participants unless stated otherwise

\begin{tabular}{|c|c|c|c|c|c|}
\hline & Mild $(n=282)$ & Moderate $(n=196)$ & Severe $(n=155)$ & Total $(n=633)$ & $P$ value \\
\hline \multicolumn{6}{|l|}{ Lacunar infarcts: } \\
\hline None & $182(64.8)$ & $95(48.5)$ & $54(35.1)$ & $331(52.5)$ & \multirow{3}{*}{$<0.001$} \\
\hline Few & $82(29.2)$ & $70(35.7)$ & $63(40.9)$ & $215(34.0)$ & \\
\hline Many & $17(6.0)$ & $31(15.8)$ & $37(24.0)$ & 85 (13.5) & \\
\hline \multicolumn{6}{|l|}{ Non-lacunar infarcts: } \\
\hline None & $264(94.3)$ & $179(91.3)$ & $130(84.4)$ & $573(91.0)$ & \multirow{2}{*}{0.001} \\
\hline At least one & $16(5.7)$ & $17(8.7)$ & $24(15.6)$ & $57(9.0)$ & \\
\hline Mean (SD) global atrophy rating & $7.5(2.4)$ & $8.0(2.4)$ & $8.8(2.2)$ & $8.0(2.4)$ & $<0.001$ \\
\hline
\end{tabular}




\section{WHAT IS ALREADY KNOWN ON THIS TOPIC}

Age related changes in white matter are often detected by neuroimaging in older people

Such changes are associated with cognitive deficits, depression, motor abnormalities, and urinary dysfunction, all of which contribute to disability in older people

\section{WHAT THIS STUDY ADDS}

Older people with extensive age related changes in white matter are at high risk of functional decline over the next three years

The annual rate of death or disability was $10.5 \%, 15.1 \%$, and $29.5 \%$, respectively, for patients with mild, moderate, or severe changes in white matter

With severe changes in white matter prognosis was only slightly worsened by incident stroke and dementia conclusive evidence has been reached as yet about interventions that could favourably alter the development of disability in such patients. Preliminary studies, however, indicate that treating hypertension might slow the progression of age related changes in white matter. ${ }^{31}$ Our data might support clinical research, helping to design preventive studies. Based on these data, therapeutic trials - for example, those experimenting on the effects of any antihypertensive agent - should include global functioning among the main outcome measures. In such studies, the risks of disability we have reported for the different severity grades of age related changes in white matter might help to define the target population and to estimate sample sizes.

We thank Patrizia Trallori for secretarial assistance throughout the LADIS study.

Participating centres and personnel

Timo Erkinjuntti, Tarja Pohjasvaara, Pia Pihanen, Raija Ylikoski, Hanna Jokinen, Meija-Marjut Somerkoski, Riitta Mäntylä, Oili Salonen (Memory Research Unit, Department of Clinical Neurosciences, Helsinki University, Helsinki, Finland); Franz Fazekas, Reinhold Schmidt, Stefan Ropele,

Brigitte Rous, Katja Petrovic, Ulrike Garmehi, Alexandra Seewann (Department of Neurology and Department of Radiology, Division of Neuroradiology, Medical University Graz, Graz, Austria); José M Ferro, Ana Verdelho, Sofia Madureira, Carla Moleiro (Serviço de Neurologia, Centro de Estudos Egas Moniz, Hospital de Santa Maria, Lisbon, Portugal); Philip Scheltens, van Straaten, Frederik Barkhof, Alida Gouw, Wiesje van der Flier (Department of Radiology and Neurology, VU Medica Centre, Amsterdam, Netherlands); Anders Wallin, Michael Jonsson, Karin Lind, Arto Nordlund, Sindre Rolstad, Ingela Isblad (Institute of Clinical Neuroscience, Goteborg University, Gothenburg, Sweden); Lars-Olof Wahlund, Milita Crisby, Anna Pettersson, Kaarina Amberla (Karolinska Institutet, Department of Neurbiology, Care Sciences and Society Karolinska University Hospital Huddinge, Sweden); Hugues Chabriat, Karen Hernandez, Annie Kurtz, Dominique Hervé, Sarah Benisty, Jean Pierre Guichard (Department of Neurology, Hopital Lariboisiere, Paris, France); Michael Hennerici, Christian Blahak, Hansjorg Baezner, Martin Wiarda, Susanne Seip (Department of Neurology, University of Heidelberg, Klinikum Mannheim, Germany); Gunhild Waldemar, Egill Rostrup, Charlotte Ryberg, Tim Dyrby, Olaf B Paulson, Ellen Garde (Memory Disorders Research Group, Department of Neurology, Rigshospitalet, and the Danish Research Centre for Magnetic Resonance Hvidovre Hospital, Copenhagen University Hospitals, Denmark); John O’Brien, Sanjeet Pakrasi, Mani Krishnan, Andrew Teodorczuk, Michael Firbank, Philip English, Thais Minett (Institute for Ageing and Health, Newcastle University, Newcastle upon Tyne).

The coordinating centre is at Department of Neurological and Psychiatric Sciences, University of Florence, Florence, Italy): Domenico Inzitari (study Coordinator); Luciano Bartolini, Anna Maria Basile, Eliana Magnani, Monica Martini, Mario Mascalchi, Marco Moretti, Leonardo Pantoni, Anna Poggesi, Giovanni Pracucci, Emilia Salvadori, Michela Simoni. The LADIS Steering Committee comprises Domenico Inzitari, (study coordinator), Timo Erkinjuntti, Philip Scheltens, Marieke Visser, Kjell Asplund (up to 2005), and Peter Langhorne (from 2005).

Contributors: DI was study coordinator and substantially contributed to the study concept, design, analysis, and interpretation of data, and writing of the paper. GP was study data manager and contributed to the analysis and interpretation of data, and writing of the paper. AP contributed to the analysis and interpretation of data, study performance, and writing of the paper. GC contributed to the study design, study performance, and writing of the paper. FB contributed to the study design, study performance, magnetic resonance imaging analysis, and writing of the paper. HC, TE, FF, JMF, MH, PL, and MCV contributed to the writing of the paper and the monitoring of the study. JO'B contributed to the study concept and design, study performance, and writing of the paper. PS contributed to the study design, study performance, and writing of the paper. L-OW, GW, AW, and LP. LP was the deputy study coordinator, and $\mathrm{Dl}$ is guarantor

Funding and sponsor: The LADIS study is supported by the European Union within the Vth European Framework Program "Quality of life and management of living resources” (1998-2002), contract No QLRT-2000- 
00446 as a concerted action. The sponsor had no role in the study design, collection, analysis, and interpretation of data, in the writing of the report, and in the decision to submit the article for publication. Competing interests: None declared.

Ethical approval: The study protocol was approved by each centre's ethics committee.

1 Pantoni L, Garcia JH. The significance of cerebral white matte abnormalities 100 years after Binswanger's report. A review. Stroke 1995;26:1293-301.

2 Breteler MMB, Van Swieten JC, Bots ML, Grobbee DE, Claus JJ, van den Hout JH, et al. Cerebral white matter lesions, vascular risk factors, and cognitive function in a population-based study: the Rotterdam study. Neurology 1994;44:1246-52.

3 Kuo HK, Lipsitz LA. Cerebral white matter changes and geriatric syndromes: is there a link? J Gerontol A Biol Sci Med Sci 2004;59:818-26.

4 Tarvonen-Schröder S, Röyttä M, Räihä I, Kurki T, Rajala T, Sourander L. Clinical features of leuko-araiosis. J Neurol Neurosurg Psychiatry 1996;60:431-6.

5 Steffens DC, Bosworth HB, Provenzale JM, MacFall JR. Subcortical white matter lesions and functional impairment in geriatric depression. Depress Anxiety 2002;15:23-8.

6 Bennett HP, Corbett AJ, Gaden S, Grayson DA, Kril JJ, Broe GA Subcortical vascular disease and functional decline: a 6-year predictor study. J Am Geriatr Soc 2002;50:1969-77.

7 Pantoni L, Basile AM, Pracucci G, Asplund K, Bogousslavsky J, Chabriat $\mathrm{H}$, et al. Impact of age-related cerebral white matter changes on the transition to disability-the LADIS study: rationale, design and methodology. Neuroepidemiology 2005;24:51-62.

8 Lawton MP, Brody EM. Assessment of older people: self-maintaining and instrumental activities of daily living. Gerontologist 1969;9:179-86.

9 Maggi S, Zucchetto M, Grigoletto F, Baldereschi M, Candelise L, Scarpini E, et al. The Italian Longitudinal Study on Aging (ILSA): design and methods. Aging (Milano) 1994;6:464-73.

10 Cox CS, Mussolino ME, Rothwell ST, Lane MA, Golden CD, Madans JH, et al. Plan and operation of the NHANES I Epidemiologic Follow up Study, 1992. Vital Health Stat 1 1997;35:1-231.

11 Van Nostrand JF, Furner SE, Suzman R. Health data on older Americans: United States 1992. Vital Health Stat 31993;27:1-5.

12 Proceedings of the CNR Workshop "Transitions in health status for older persons-international comparisons." Rome: International Network of the Methodology of Longitudinal Studies of Aging, October 1998.

13 American Psychiatric Association. Diagnostic and statistical manual of mental disorders. 4th ed. Washington, DC: American Psychiatric Association, 1994.

14 Hatano S. Experience from a multicentre stroke register: a preliminary report. Bull World Health Organ 1976;54:541-53.

15 Fazekas F, Chawluk JB, Alavi A, Hurtig HI, Zimmerman RA. MR signal abnormalities at 1.5T in Alzheimer's dementia and normal aging. AJNR Am J Neuroradiol 1987;8:421-6.

16 Van Straaten ECW, Fazekas F, Rostrup E, Scheltens P, Schmidt R, Pantoni L, et al. Impact of white matter hyperintensities scoring method on correlations with clinical data: the LADIS study. Stroke 2006;37:836-40.

17 Van der Flier WM, van Straaten EC, Barkhof F, Verdelho A, Madureira S, Pantoni L, et al. Small vessel disease and general cognitive function in nondisabled elderly: the LADIS study. Stroke 2005;36:2116-20.

18 Ryberg C, Rostrup E, Sjöstrand K, Paulson OB, Barkhof F, Scheltens P, et al. White matter changes contribute to corpus callosum atrophy in the elderly: the LADIS study. AJNR Am I Neuroradiol 2008;29:1498-504.

19 Stuck AE, Walthert JM, Nikolaus T, Bula CJ, Hohmann C, Beck JC. Risk factors for functional status decline in community-living elderly people: a systematic literature review. Soc Sci Med 1999;48:445-69.

20 Inzitari D. Leukoaraiosis: an independent risk factor for stroke? Stroke 2003;34:2067-71

21 Inzitari D, Simoni M, Pracucci G, Poggesi A, Basile AM, Chabriat H, et al. Risk of rapid global functional decline in elderly patients with severe cerebral age-related white matter changes: the LADIS study. Arch Intern Med 2007;167:81-8.

22 Katz S. Assessing self-maintenance: activities of daily living, mobility, and instrumental activities of daily living. J Am Geriatr Soc 1983;31:721-7.

23 Avlund K. Methodological challenges in measurements of functional ability in gerontological research. A review. Aging (Milano) 1997;9:164-74

24 Nagi S. Some conceptual issues in disability and rehabilitation. In: Sussman MB, ed. Sociology and rehabilitation. Washington, DC: American Sociological Association, 1965:100-13.

25 Rosano C, Kuller LH, Chung H, Arnold AM, Longstreth WT Jr, Newman AB. Subclinical brain magnetic resonance imaging abnormalities predict physical functional decline in high-functioning older adults. J Am Geriatr Soc 2005;53:649-54.

26 Prins ND, van Dijk EJ, den Heijer T, Vermeer SE, Koudstaal PJ, Oudkerk M, et al. Cerebral white matter lesions and the risk of dementia. Arch Neurol 2004;61:1531-4.

27 Prins ND, van Dijk EJ, den Heijer T, Vermeer SE, Jolles J, Koudstaal PJ, et al. Cerebral small-vessel disease and decline in information processing speed, executive function and memory. Brain 2005;128:2034-41.

28 Pantoni L, Garcia JH. Pathogenesis of leukoaraiosis: a review. Stroke 1997;28:652-9.

29 Fernando MS, Simpson JE, Matthews F, Brayne C, Lewis CE, Barber R, et al. White matter lesions in an unselected cohort of the elderly: molecular pathology suggests origin from chronic hypoperfusion injury. Stroke 2006;37:1391-8.

30 Schmidt R, Scheltens P, Erkinjuntti T, Pantoni L, Markus HS, Wallin A, et al. White matter lesion progression: a surrogate endpoint for trials in cerebral small-vessel disease. Neurology 2004;63:139-44.

31 Dufouil C, Chalmers J, Coskun O, Besancon C, Bousser MG, Guillon P, et al. Effects of blood pressure lowering on cerebral white matter hyperintensities in patients with stroke: the PROGRESS (Perindopril Protection Against Recurrent Stroke Study) MRImaging Substudy. Circulation 2005;112:1644-50.

Accepted: 13 February 2009 